\title{
Development and internal validation of a model predicting severe maternal morbidity using variables available pre-conception and in early pregnancy: a population-based study
}

Natalie Dayan ${ }^{1}$, Gabriel Shapirio ${ }^{2}$, Jin Luo ${ }^{3}$, Jun Guan ${ }^{4}$, Deshayne Fell ${ }^{5}$, Carl Laskin ${ }^{6}$, Olga Basso ${ }^{2}$, Alison Park ${ }^{4}$, and Joel Ray ${ }^{7}$

${ }^{1}$ McGill University Health Centre

${ }^{2}$ McGill University

${ }^{3}$ Institute for Clinical Evaluative Sciences

${ }^{4} \mathrm{ICES}$

${ }^{5}$ University of Ottawa

${ }^{6}$ University of Toronto

${ }^{7}$ Departments of Medicine and Obstetrics and Gynaecology, St. Michael's Hospital, University of Toronto, Ontario, Canada

December 27, 2020

\begin{abstract}
Objective: To improve the prediction of maternal end-organ injury or death using routinely-collected variables from the prepregnancy and the early pregnancy period. Design: Population-based cohort study using linked administrative health data. Setting: Ontario, Canada, April 1, 2006 to March 31, 2014. Sample: Women aged 18-60 years with a livebirth or stillbirth, of which one birth was randomly selected per woman. Methods and main outcome measures: We constructed a CPM for the primary composite outcome of any maternal end-organ injury or death, arising between 20 weeks' gestation and 42 days after the birth hospital discharge date. Our CPM included variables collected from 12 months before estimated conception until 19 weeks' gestation. We developed a separate CPM for parous women to allow for the inclusion of factors from previous pregnancy(ies). Results: Of 634,290 women, 1969 experienced the primary composite outcome (3.1 per 1000). Predictive factors in the main CPM included maternal world region of origin, chronic medical conditions, parity, and obstetrical/perinatal issues - with moderate model discrimination (C-statistic 0.68, 95\% CI 0.66-0.69). Among 333,435 parous women, the C-statistic was $0.71(0.69-0.73)$ in the model using variables from the current (index) pregnancy as well as pre-pregnancy predictors and variables from any previous pregnancy. Conclusions: A combination of factors ascertained early in pregnancy through a basic medical history help to identify women at risk for severe morbidity, who may benefit from targeted preventive and surveillance strategies including appropriate specialty-based antenatal care pathways. Further refinement of this model would enable clinical use.
\end{abstract}

Title: Development and internal validation of a model predicting severe maternal morbidity using variables available pre-conception and in early pregnancy: a population-based study

Natalie DAYAN MD ${ }^{1,2}$, Gabriel D SHAPIRO $\mathrm{PhD}^{1,2}$, Jin LUO $\mathrm{MSc}^{3}$, Jun GUAN MSc ${ }^{3}$, Deshayne B FELL $\mathrm{PhD}^{3,4}$, Carl A LASKIN MD ${ }^{5,6}$, Olga BASSO PhD ${ }^{2,7}$, Alison L PARK MSc ${ }^{3}$, Joel G RAY MD ${ }^{3,5}$

1. Department of Medicine and Research Institute, McGill University Health Centre, Montreal, Quebec, Canada 
2. Department of Epidemiology, Biostatistics and Occupational Health, McGill University, Montreal, Quebec, Canada

3. ICES, Toronto, Ontario, Canada

4. School of Epidemiology and Public Health, University of Ottawa, Ottawa, Ontario, Canada

5. Department of Medicine and Obstetrics and Gynecology, University of Toronto

6. TRIO Fertility, Toronto, Ontario, Canada

7. Department of Obstetrics and Gynecology, McGill University Health Centre, Montreal, Quebec, Canada

Address for corresponding author:

Dr. Natalie Dayan

Centre for Outcomes Research and Evaluation (CORE)

McGill University Health Centre

5252 de Maisonneuve West, 2B.40

Montréal QC H4A 3S5

Natalie.Dayan@mcgill.ca

T: 514-934-1934 x 76125

ORCiD iD: 0000-0002-2112-1253

Running title: Prediction of severe maternal morbidity

\section{Abstract}

Objective: To improve the prediction of maternal end-organ injury or death using routinely-collected variables from the pre-pregnancy and the early pregnancy period.

Design: Population-based cohort study using linked administrative health data.

Setting: Ontario, Canada, April 1, 2006 to March 31, 2014.

Sample: Women aged 18-60 years with a livebirth or stillbirth, of which one birth was randomly selected per woman.

Methods and main outcome measures: We constructed a CPM for the primary composite outcome of any maternal end-organ injury or death, arising between 20 weeks' gestation and 42 days after the birth hospital discharge date. Our CPM included variables collected from 12 months before estimated conception until 19 weeks' gestation. We developed a separate CPM for parous women to allow for the inclusion of factors from previous pregnancy(ies).

Results: Of 634,290 women, 1969 experienced the primary composite outcome (3.1 per 1000). Predictive factors in the main CPM included maternal world region of origin, chronic medical conditions, parity, and obstetrical/perinatal issues - with moderate model discrimination (C-statistic 0.68, 95\% CI 0.66-0.69). Among 333,435 parous women, the C-statistic was 0.71 (0.69-0.73) in the model using variables from the current (index) pregnancy as well as pre-pregnancy predictors and variables from any previous pregnancy.

Conclusions: A combination of factors ascertained early in pregnancy through a basic medical history help to identify women at risk for severe morbidity, who may benefit from targeted preventive and surveillance strategies including appropriate specialty-based antenatal care pathways. Further refinement of this model would enable clinical use.

Tweetable abstract: Clinical factors ascertained through a medical history in early pregnancy can moderately predict severe maternal morbidity. 
Funding: This study was supported by funding from the Canadian Institutes of Health Research (grant number 15139).

Keywords: Women's health, Epidemiology, Obstetrics and Gynaecology

\section{Introduction}

Prediction of severe maternal morbidity has been identified as a critical research gap in obstetrics. ${ }^{1}$ Industrialized countries such as Canada and the UK experience similar low levels of maternal mortality, necessitating a shift in focus on 'near miss' events as a means to improving the health and quality of care for pregnant women. ${ }^{2}$ Many maternal characteristics are known pre-conception or early in pregnancy and are strong risk factors for the development of severe maternal morbidity. ${ }^{3,4}$ Therefore, a combination of such factors may reliably predict its onset, enabling evidence-based and rational early triage of high-risk women for enhanced surveillance and subspecialty-based care.

Advances in maternal morbidity risk prediction include a US obstetric comorbidity index,${ }^{5}$ which was externally validated within a Canadian population, resulting in modest discrimination (C-statistic of 0.66, 95\% confidence interval [CI] 0.65-0.67). ${ }^{6}$ That index included variables that both preceded, and were simultaneous with, the onset of severe maternal morbidity, making it a useful research tool for identifying the burden of morbidity but less so for clinical prediction. Others have developed models focused on specific subtypes of maternal morbidity, such as cardiovascular-related conditions. ${ }^{7}$ Models predicting maternal mortality include the Collaborative Integrated Pregnancy High-dependency Estimate of Risk (CIPHER) model (C-statistic 0.82, 95\% CI 0.81-0.84) and the Maternal Severity Index (C-statistic 0.83, 95\% CI 0.80-0.85), ${ }^{8}$ both developed among women either already critically ill or hospitalized, and mostly later in gestation.

Since severe maternal morbidity predominantly arises around birth or early postpartum, ${ }^{9}$ the ideal timeframe for prediction is before or early in pregnancy to facilitate effective preventive strategies such as referral to highrisk centres or shared-care antenatal care pathways. ${ }^{10,11}$ Existing models do not enable these latter steps, nor do they account for important pre-pregnancy factors, such as maternal infertility and its treatment, which are associated with severe maternal morbidity. ${ }^{12}$ Additionally, existing prediction efforts did not consider prior adverse pregnancy outcomes among parous women. We therefore undertook the current study to develop and internally validate a clinical prediction model $(\mathrm{CPM})$ of severe maternal morbidity, defined as acute end-organ injury or death, using readily available factors ascertained pre-pregnancy and prior to 20 weeks' gestation in a population-based study in Ontario - Canada's most populous and multi-ethnic province.

\section{Methods}

The use of data in this project was authorized under section 45 of Ontario's Personal Health Information Protection Act, which does not require review by a Research Ethics Board. We followed the Transparent reporting of a multivariable prediction model for individual prognosis or diagnosis (TRIPOD) guideline for reporting of prediction studies. ${ }^{13}$

\section{Population and data sources}

All women with a pregnancy lasting beyond 20 weeks' gestation, and who delivered within an Ontario hospital between April 1, 2006 and March 31, 2014, were identified within the Better Outcomes Registry \& Network (BORN) databases. ${ }^{14}$ Data beyond 2014 were not available in these datasets. The BORN registry captures over $99 \%$ of hospital births in the province, and has been validated for data completeness and accuracy. ${ }^{15,16}$ We used the Registered Persons Database, the Immigration, Refugees and Citizenship Canada's Permanent Resident Database, the Ontario Health Insurance Plan (OHIP) outpatient claims database, and the Canadian Institute for Health Information (CIHI) Discharge Abstract Database to capture maternal demographics, pre-existing health conditions and diagnoses and procedures documented during a hospitalization (Table S1 for variables and diagnostic codes used to develop the study cohort). The datasets were linked using unique encoded identifiers and analysed at ICES- a not-for-profit provincial research entity that houses a large network of health administrative databases (https://www.ices.on.ca/). 
We excluded ectopic pregnancies, pregnancies resulting in abortion or miscarriage or ending before 20 weeks' gestation. We randomly sampled one birth (live- or stillbirth) per woman to avoid potential correlations among pregnancies of multiparous women (Table S1; Figure S1).

\section{Study outcomes}

The primary composite outcome was maternal end-organ injury or death arising between 20 weeks' gestation and up to 42 days after the index birth hospital discharge date. The list of conditions used to define maternal end-organ injury was based on the CPM developed by Bateman ${ }^{5}$ and validated by Metcalfe, ${ }^{6}$ comprising 20 diagnoses and procedures, and consistent with Canadian perinatal surveillance definitions for severe maternal morbidity and death. ${ }^{17-19}$ (Table S1).

A secondary outcome was all-cause maternal mortality, from birth until 365 days postpartum, since previous work has shown a persistent increase in mortality risk beyond the early postpartum period..$^{20,21}$

Candidate predictors, variable selection, and coding

Demographic, medical and obstetric factors known to be associated with an increased risk of severe maternal morbidity were considered as candidate predictors. These included: estimated maternal age at conception (continuous, categorical, and squared terms); residential income quintile; world region of origin (Table S2); attendance at a first-trimester prenatal care visit; pre-pregnancy body mass index (BMI); parity; multiple gestation; infertility; infertility treatment; placental disorders (e.g., placenta praevia, placenta accreta); and pre-existing medical conditions coded within 12 months before the estimated date of conception (Table S1). Substantial missing data was noted only for the variable pre-pregnancy BMI $(63.79 \%)$. We tested models in which BMI was modelled as a continuous variable and where missing values were assigned the median BMI $\left(24.2 \mathrm{~kg} / \mathrm{m}^{2}\right)$. We further tested models in which BMI was divided into the following categories: $<18.5$ $\mathrm{kg} / \mathrm{m}^{2}, 18.5-24.9 \mathrm{~kg} / \mathrm{m}^{2}$ (reference category), $25-29.9 \mathrm{~kg} / \mathrm{m}^{2},>30 \mathrm{~kg} / \mathrm{m}^{2}$, and missing. Certain categorical variables with a low frequency in the cohort were combined with other similar variables (e.g., pre-existing cardiovascular conditions; placental conditions and anomalies). Variables were also assessed for collinearity by checking the variance inflation factor (VIF), and where collinear (VIF > 5), the most commonly reported variable was selected. ${ }^{22}$

In the CPM restricted to the sub-cohort of parous women, in addition to the above variables, we included complications coded in any previous pregnancy as predictors (Table S1).

Possible interactions between variables were assessed and included if statistically significant at alpha $=0.10 .^{23}$

Statistical analysis

\section{Descriptive statistics}

We used standardized differences to contrast births with and without maternal end-organ injury or death, with a value $>0.10$ indicating an important difference in baseline characteristics. ${ }^{24}$

\section{Model discrimination}

Among the entire cohort, a logistic regression model was fit using the final selected variables to predict maternal acute end-organ injury or death from 20 weeks' gestation until 42 days postpartum. A backward elimination method was applied for variable selection, with predictor evaluation based on a balance of the model's C-statistic, clinical influence, and statistical significance. For continuous predictor variables such as age in which non-linear associations with the outcome were observed, a quadratic (squared) term was added to the model. Model discrimination was expressed as a C-statistic and its $95 \% \mathrm{CI}$, as well as visual detection of a receiver operating curve (ROC). We considered a C-statistic of $<0.5$ to be not useful, 0.5 to 0.6 poor, 0.6 to 0.7 moderate, and [?] 0.7 as good. ${ }^{25}$

\section{Model internal validation}


To arrive at an optimism-corrected C-statistic, we used a bootstrapping approach, with 500 bootstrap samples selected from the original cohort, with replacement ${ }^{26}$ - an approach known to produce stable estimates with low risk of bias. ${ }^{27}$ The optimism-corrected C-statistic was defined as the C-statistic from the original data minus the optimism value. ${ }^{28}$

\section{Model calibration}

Model calibration was assessed by visual inspection of calibration plots of observed vs. expected probabilities of the outcome, where a 45-degree line denotes good calibration, and a slope of 1 indicates perfect agreement between observed and expected events. ${ }^{29}$

\section{Risk classification}

We used a risk classification table and computed likelihood ratios (LRs) ${ }^{30}$ with associated $95 \%$ CI to assess the main model's ability to stratify the population as low or high-risk. We divided the population into five groups of predicted probability: very low risk $(<1.5$ per 1000), low risk (1.5 to 3 per 1,000), intermediate risk $(3$ to 5 per 1,000$)$, high risk ( 5 to 15 per 1,000$)$ and very high risk $(>15$ per 1,000$)$. These cut-offs were chosen based on the overall incidence of our primary outcome of 3.1 per 1,000 which we assumed to reflect the risk among the majority of the cohort. Positive LRs of $>5$ and $>10$ were interpreted as moderately or very useful "rule-in" tests, while values between 0.2 and 0.5 , and $<0.1$ were considered moderately and very useful "rule-out" tests. ${ }^{31}$

\section{Funding}

This study was supported by funding from the Canadian Institutes of Health Research (grant number 15139).

\section{Results}

After sampling one birth per woman from among 853,517 eligible births, the total cohort comprised 634,290 births (Figure S1). The primary outcome of end-organ injury or death from 20 weeks' gestation up to 42 days postpartum occurred in 1,969 women (3.1 per 1,000), including 62 deaths ( 0.1 per 1,000). Women who experienced the primary outcome were older, more likely to have a pre-existing medical condition, and to have had infertility treatment (Table S3).

The most frequent factors contributing to end-organ injury or death were acute heart failure (40.6\%), need for assisted ventilation (29.2\%), acute renal failure (12.0\%) and shock (10.1\%) (Table 1).

\section{Model discrimination and internal validation}

\section{Overall cohort}

In the overall cohort $(n=634,290)$, variables significantly associated with end-organ injury or death included maternal age, low income, world region of origin, high BMI, pre-existing medical conditions, and placental disorders (Table S4), which contributed to the final model. Attendance at a first-trimester antenatal visit and parity were inversely associated with the composite outcome. The corresponding model C-statistic was 0.68 (95\% CI 0.66-0.69) (Figure 1). There was minimal overfitting of the model, with mean optimism of 0.0055 (95\% CI 0.0050-0.0061), and an optimism-corrected C-statistic of 0.67 (95\% CI 0.66-0.68). Model discrimination was unchanged when BMI was included. We tested 300 pairwise interactions, of which 13 interactions were statistically significant. The main model including interaction terms resulted in similar model discrimination as the main model (C-statistic 0.69, 95\% CI 0.68-0.70), however this model included unstable estimates. Therefore, the model without interaction terms was chosen as the most balanced and efficient model.

All-cause mortality from birth until 365 days postpartum occurred in 194 women over the study time period $(0.3$ per 1,000). The final multivariable model for all-cause mortality no longer retained world region of origin, parity, previous spontaneous abortion, and several medical comorbidities (Table S5). Major psychiatric conditions and alcohol and substance use newly emerged as predictors. The corresponding C-statistic was 
0.70 (95\% CI 0.66-0.74) (Figure S2). However, this model was slightly over-fitted, and the optimism-corrected C-statistic was 0.67 (95\% CI 0.63-0.71).

The risk classification table for the main model, dividing the cohort according to the five categories of predicted risk of acute end-organ injury or death (Table 2) demonstrated the capacity of this model to classify women who are at very low risk (-LR 0.41, 95\% CI 0.33-0.52) and those at very high risk of the outcome (+LR 8.58, 95\% CI 7.32-10.05), but was less useful in classifying women in intermediate risk categories.

\section{Sub-cohort of parous women}

In the sub-cohort of 333,435 parous women, the aforementioned variables significantly associated with endorgan injury or death persisted, as did the addition of an unplanned caesarean delivery and severe organ injury in a previous birth (Table S6). The C-statistic was 0.61 (95\% CI 0.59-0.63) when limited to variables from the index pregnancy (Figure 2a), rising to 0.69 (95\% CI 0.67-0.70) after adding pre-pregnancy predictors (Figure $2 \mathrm{~b}$ ), and 0.71 (95\% CI 0.69-0.73) when including the variables from a previous pregnancy (Figure 2c). We noted minimal overfitting for each CPM, with optimism-corrected C-statistics of 0.60 (95\% CI 0.58-0.62), 0.68 (95\% CI 0.66-0.70), and 0.70 (95\% CI 0.69-0.72), respectively.

\section{Model fit and calibration}

Visual inspection of the calibration plots in the entire cohort suggested good agreement between observed and expected events for the primary outcome, with slightly worse calibration for mortality (Figure S3a-b). Among parous women, model calibration for maternal end-organ injury or death improved from the base model to models including variables measured pre-pregnancy and in a previous pregnancy (Figure S3c-e).

\section{Discussion}

\section{Main findings}

We have shown that a CPM based on pre-pregnancy and early pregnancy variables can moderately discriminate women destined for a severely morbid event or death from those likely to have uncomplicated pregnancies. The inclusion of prior pregnancy factors slightly enhanced these metrics. Our CPM displayed good calibration, indicating that a combination of routinely measured pre-pregnancy and early pregnancy factors can estimate the absolute risk of acute end-organ injury or death with reasonable accuracy. Using this CPM effectively increased the probability of identifying a very high-risk woman with this outcome by $40 \%$, and reduced the probability in someone considered very low-risk by $20 \%{ }^{31}$, but was less useful in classifying women in intermediate risk categories. This suggests that additional clinical, laboratory, or paraclinical factors are needed to accurately predict morbidity in all women, and further, that a certain proportion of these events are truly sudden and unpredictable.

\section{Strengths and limitations}

The CPMs in this study relied on information that is routinely known at the time of the first antenatal visit, using variables that were temporally remote from when most maternal morbid events arise - largely around the time of birth. ${ }^{9}$ Moreover, our source population comprised all pregnancies from gestational week 20. However, our datasets had few routinely collected clinical measures, such as blood pressure and haemoglobin or glucose concentrations, or first-trimester screening biomarkers. In the prediction of preterm preeclampsia, for example, a model that contained a combination of clinical and paraclinical variables (including placental biomarkers) performed better than with either set of variables in isolation. ${ }^{32}$ BMI was incomplete in our dataset, as is common in most administrative data sources. However, the proportion in any given BMI category and those with missing values was not appreciably different among women with and without the outcome. Thus, while the contribution of BMI to the outcome may thus not have been well represented in our models, this unlikely changed the overall model performance.

Prediction models are often used to estimate an individual's absolute risk of a serious adverse event that might be mitigated with the use of a particular therapy, while avoiding subjecting individuals at low predicted 
risk to potential harmful effects of such therapy. ${ }^{33}$ In obstetrics, serious adverse events are rare, with limited options for targeted prevention. We acknowledge, therefore, the limitations afforded by the C-statistic to discriminate between individuals with and without a rare adverse event, in which a high false positive rate might be justified. ${ }^{34}$ The LRs add clinical meaning to the CPM and serve as a foundation for what might be considered reasonable predictability of rare but catastrophic obstetric events.

\section{Interpretation}

The CPMs in this study relied on information that is routinely known at the time of the first antenatal visit, and that is temporally remote from when most morbid events arise - around the time of birth..$^{9}$ Our main CPM shows the potential utility of harnessing data in early pregnancy to predict a variety of later adverse maternal outcomes. Consistent with previous research on postnatal mortality, ${ }^{21}$ our CPM for allcause mortality showed substance use, alcohol use, and psychiatric conditions to be significant predictors of death up to 365 days postpartum.

Severe maternal morbidity rates have stagnated within Western nations, yet evidence-based strategies to reduce their burden are lacking. ${ }^{9}$ With further refinement of this CPM, a clinical risk calculator could be developed to help triage women for enhanced surveillance or referral to subspecialty care or shared-care antenatal pathways - decisions that at present rely principally on clinical judgment. The development and refinement of future CPMs for severe maternal morbidity should consider adding first-trimester placental biomarkers and other maternal biomarkers alongside routinely measured clinical variables, such as blood pressure and weight. The availability of such variables might facilitate prediction of the whole of severe morbidity as well as cause-specific outcomes, and better inform individualized and targeted prevention. ${ }^{35}$

\section{Conclusion}

In conclusion, a CPM developed using pre-pregnancy and early pregnancy predictors available within administrative datasets had moderate prediction of maternal acute end-organ injury or death. The addition of factors from a prior pregnancy among parous women slightly improved the CPM performance. Enhancement of these CPMs, using direct clinical measures, and by external validation or using machine learning, is needed.

Acknowledgements: Dr. Dayan would like to acknowledge Dr. Ugochinyere Ukah for review of the manuscript and suggestions for model refinement.

Disclosure of Interests: The authors declare they have no conflicts of interest.

Contribution to Authorship: ND, AP and JGR designed the study and interpreted analyses. ND wrote the manuscript. JL and JG assisted with study design and cohort creation and performed data analyses. GDS contributed to study design and data analysis interpretation. DBF, CL, OB, and AP assisted with study design and data interpretation. All authors revised the manuscript critically for important intellectual content, approved the version to be published and agreed to be accountable for all aspects of the work.

Detail of ethics approval: The use of data in this project was authorized under section 45 of Ontario's Personal Health Information Protection Act, which does not require review by a Research Ethics Board.

Funding: This study was supported by funding from the Canadian Institutes of Health Research (grant number 15139). The funder had no role in the design and conduct of the study; collection, management, analysis, and interpretation of the data; preparation, review, or approval of the manuscript; and decision to submit the manuscript for publication. Dr. Dayan is supported by the Fondation de Recherche du QuebecSante (FRQS) Chercheur Boursier Clinicien award. This study was also supported by ICES, which is funded by an annual grant from the Ontario Ministry of Health and Long-Term Care (MOHLTC). Parts of this material are based on data and information compiled and provided by MOHLTC and CIHI. The analyses, conclusions, opinions and statements expressed herein are solely those of the authors and do not reflect those of the funding or data sources; no endorsement is intended or should be inferred. This Study is based in part on data provided by Better Outcomes Registry and Network ("BORN"), part of the Children's Hospital of 
Eastern Ontario. The interpretation and conclusions contained herein do not necessarily represent those of BORN Ontario.

\section{References}

1. D'Alton ME, Bonanno CA, Berkowitz RL, et al. Putting the "M" back in maternal-fetal medicine. Am J Obstet Gynecol.2013;208(6):442-448.

2. van Roosmalen J, Zwart J. Severe acute maternal morbidity in high-income countries. Best Pract Res Clin Obstet Gynaecol.2009;23(3):297-304.

3. Aoyama K, Pinto R, Ray JG, et al. Association of Maternal Age With Severe Maternal Morbidity and Mortality in Canada. JAMA Netw Open. 2019;2(8):e199875.

4. Lindquist A, Knight M, Kurinczuk JJ. Variation in severe maternal morbidity according to socioeconomic position: a UK national case-control study. BMJ Open. 2013;3(6).

5. Bateman BT, Mhyre JM, Hernandez-Diaz S, et al. Development of a comorbidity index for use in obstetric patients. Obstet Gynecol.2013;122(5):957-965.

6. Metcalfe A, Lix LM, Johnson J-A, Currie G, Lyon AW, Bernier F, et al. Validation of an obstetric comorbidity index in an external population.British Journal of Obstetrics $\&$ Gynaecology. 2015;122:17481755 .

7. Malhame I, Danilack VA, Raker CA, et al. Cardiovascular severe maternal morbidity in pregnant and postpartum women: development and internal validation of risk prediction models. BJOG. 2020 .

8. Aoyama K, D'Souza R, Pinto R, et al. Risk prediction models for maternal mortality: A systematic review and meta-analysis. PLoS One. 2018;13(12):e0208563.

9. Ray JG, Park AL, Dzakpasu S, et al. Prevalence of Severe Maternal Morbidity and Factors Associated With Maternal Mortality in Ontario, Canada. JAMA Netw Open. 2018;1(7):e184571.

10. Menard MK. Toward Achieving Risk-Appropriate Maternity Care: Maternal Morbidity Prediction. Obstet Gynecol.2019;134(2):213-215.

11. Macones GA. Understanding and reducing serious maternal morbidity: a step in the right direction. Obstet Gynecol. 2013;122(5):945-946.

12. Dayan N, Joseph KS, Fell DB, et al. Infertility treatment and risk of severe maternal morbidity: a propensity score-matched cohort study. CMAJ. 2019;191(5):E118-E127.

13. Collins GS, Reitsma JB, Altman DG, Moons KG. Transparent reporting of a multivariable prediction model for individual prognosis or diagnosis (TRIPOD): the TRIPOD statement. BMJ. 2015;350:g7594.

14. BORN Ontario. Legacy Data Elements. https://www.bornontario.ca/en/data/legacy-dataelements.aspx. Published 2019. Accessed 13 September, 2019.

15. Dunn S, Lanes A, Sprague AE, et al. Data accuracy in the Ontario birth Registry: a chart re-abstraction study. BMC Health Serv Res. 2019;19(1):1001.

16. Dunn S, Bottomley J, Ali A, Walker M. 2008 Niday Perinatal Database quality audit: report of a quality assurance project. Chronic Dis Inj Can. 2011;32(1):32-42.

17. Joseph KS, Liu S, Rouleau J, Kirby RS, Kramer MS, Sauve R, et al. Severe Maternal Morbidity in Canada, 2003 to 2007: Surveillance Using Routine Hospitalization Data and ICD-10CA Codes. Journal of Obstetrics 83 Gynaecology Canada: JOGC. 2010;32(9):837-846.

18. Main EK, Abreo A, McNulty J, et al. Measuring severe maternal morbidity: validation of potential measures. Am J Obstet Gynecol.2016;214(5):643 e641-643 e610. 
19. Roberts CL, Cameron CA, Bell JC, Algert CS, Morris JM. Measuring maternal morbidity in routinely collected health data: development and validation of a maternal morbidity outcome indicator. Med Care.2008;46(8):786-794.

20. Grigoriadis S, Wilton AS, Kurdyak PA, et al. Perinatal suicide in Ontario, Canada: a 15-year populationbased study. CMAJ.2017;189(34):E1085-E1092.

21. Ray JG, Zipursky J, Park AL. Injury-related maternal mortality.Am J Obstet Gynecol. 2018;219(3):307308.

22. Hair JF, Black WC, Babin BJ, Anderson RE, Tatham RLMultivariate data analysis. Upper Saddle River, NJ: Pearson Prentice Hall; 2006.

23. Steyerberg EW. Clinical prediction models: A practical approach to development, validation, and updating. In: Links S, ed. New York, NY2009.

24. Austin PC. Using the standardized difference to compare the prevalence of a binary variable between two groups in observational research. Communications in Statistics - Simulation and Computation. 2009;38.

25. Hanley JA, McNeil BJ The meaning and use of the area under a receiver operating characteristic (ROC) curve. Radiology.1982;143(1):29.

26. Steyerberg EW. Mathematics and Statistics. Clinical prediction models: A practical approach to development, validation, and updating.New York, NY: Springer; 2009.

27. Steyerberg EW, Harrell FE, Jr., Borsboom GJ, Eijkemans MJ, Vergouwe Y, Habbema JD. Internal validation of predictive models: efficiency of some procedures for logistic regression analysis. J Clin Epidemiol. 2001;54(8):774-781.

28. Harrell FE, Jr., Lee KL, Mark DB. Multivariable prognostic models: issues in developing models, evaluating assumptions and adequacy, and measuring and reducing errors. Stat Med. 1996;15(4):361-387.

29. Steyerberg EW, Vickerrs AJ, Cook NR, Gerds T, Gonen M, Obuchowski N, et al. Assessing the performance of prediction models: a framework for some traditional and novel measures. Epidemiology.2010;21:128138.

30. Deeks JJ, Altman DG Diagnostic tests 4: Likelihood ratios. .British Medical Journal. 2004;329.

31. McGee S. Simplfying Likelihood Ratios. J Gen Intern Med.2002;17(8):647-650.

32. Akolekar R, Syngelaki A, Poon L, Wright D, Nicolaides KH. Competing risks model in early screening for preeclampsia by biophysical and biochemical markers. Fetal Diagn Ther. 2013;33(1):8-15.

33. Alba AC, Agoritsas T, Walsh M, et al. Discrimination and Calibration of Clinical Prediction Models: Users' Guides to the Medical Literature.JAMA. 2017;318(14):1377-1384.

34. Romero-Brufau S, Huddleston JM, Escobar GJ, Liebow M. Why the C-statistic is not informative to evaluate early warning scores and what metrics to use. Critical care (London, England). 2015;19:285.

35. Davidson AJ, Park AL, Ray JG. Navigating severe maternal morbidity using big data: Green, yellow, and red flags for researchers. Obstet Med. 2019;12(3):105-106.

Table 1. Occurrence of maternal end-organ injury or death between 20 weeks' gestation and up to 42 days after birth, and the ranking of the most prevalent morbidity indicators 


\begin{tabular}{|c|c|c|c|}
\hline Outcome & $\begin{array}{l}\text { Number of outcome } \\
\text { events }\end{array}$ & Rate per 1000 & $\begin{array}{l}\text { Proportion of all } \\
\text { outcomes (\%) (a) }\end{array}$ \\
\hline $\begin{array}{l}\text { Maternal end-organ } \\
\text { injury or death } \\
\text { between } 20 \text { weeks' } \\
\text { gestation and } 42 \text { days } \\
\text { after birth }\end{array}$ & 1969 & 3.1 & 100.0 \\
\hline $\begin{array}{l}\text { Maternal end-organ } \\
\text { injury, without death, } \\
\text { between } 20 \text { weeks' } \\
\text { gestation and } 42 \text { days } \\
\text { after birth }\end{array}$ & 1907 & 3.0 & 96.9 \\
\hline $\begin{array}{l}\text { Death between } 20 \\
\text { weeks' gestation and } 42 \\
\text { days after birth }\end{array}$ & 62 & 0.1 & 3.1 \\
\hline $\begin{array}{l}\text { Death without } \\
\text { end-organ injury }\end{array}$ & 19 & 0.03 & 1.0 \\
\hline $\begin{array}{l}\text { Combined maternal } \\
\text { end-organ injury and } \\
\text { death between } 20 \\
\text { weeks' gestation and } 42 \\
\text { days after birth }\end{array}$ & 43 & 0.07 & 2.2 \\
\hline Acute heart failure & 800 & 1.3 & 40.6 \\
\hline $\begin{array}{l}\text { Assisted ventilation } \\
\text { through endotracheal } \\
\text { tube }\end{array}$ & 575 & 0.9 & 29.2 \\
\hline Acute renal failure & 237 & 0.4 & 12.0 \\
\hline Shock & 198 & 0.3 & 10.1 \\
\hline $\begin{array}{l}\text { Adult respiratory } \\
\text { distress syndrome or } \\
\text { respiratory failure }\end{array}$ & 157 & 0.3 & 8.0 \\
\hline $\begin{array}{l}\text { Puerperal } \\
\text { cerebrovascular } \\
\text { disorders }\end{array}$ & 134 & 0.2 & 6.8 \\
\hline Acute liver disease & 85 & 0.1 & 4.3 \\
\hline $\begin{array}{l}\text { Disseminated } \\
\text { intravascular } \\
\text { coagulation }\end{array}$ & 54 & 0.09 & 2.7 \\
\hline $\begin{array}{l}\text { Acute } \\
\text { psychosis/delirium }\end{array}$ & 49 & 0.08 & 2.5 \\
\hline Dialysis & 37 & 0.06 & 1.9 \\
\hline Sepsis & 33 & 0.05 & 1.7 \\
\hline $\begin{array}{l}\text { Acute myocardial } \\
\text { infarction }\end{array}$ & 32 & 0.05 & 1.6 \\
\hline Left ventricular failure & 32 & 0.05 & 1.6 \\
\hline Status epilepticus & 23 & 0.04 & 1.2 \\
\hline Status asthmaticus & 22 & 0.03 & 1.1 \\
\hline $\begin{array}{l}\text { Cerebral oedema or } \\
\text { coma }\end{array}$ & 20 & 0.03 & 1.0 \\
\hline
\end{tabular}




\begin{tabular}{llll}
\hline Outcome & $\begin{array}{l}\text { Number of outcome } \\
\text { events }\end{array}$ & Rate per 1000 & $\begin{array}{l}\text { Proportion of all } \\
\text { outcomes (\%) }\end{array}$ \\
\hline $\begin{array}{l}\text { Assisted ventilation } \\
\text { through tracheostomy }\end{array}$ & 8 & 0.01 & 0.4 \\
\hline
\end{tabular}

(a) Categories not mutually exclusive

Table 2. Risk classification comparing predicted and observed risks of the outcome using five groups of predicted probability, and associated likelihood ratios in each group. Data are from main model predicting acute end organ injury or death from 20 days gestation until 42 days after birth $(\mathrm{n}=634,290)$

\begin{tabular}{|c|c|c|c|c|}
\hline $\begin{array}{l}\text { Predicted risk } \\
\text { group (per } 1,000)\end{array}$ & $\begin{array}{l}\text { Observed acute } \\
\text { end-organ injury } \\
\text { or death }\end{array}$ & $\begin{array}{l}\text { Observed acute } \\
\text { end-organ injury } \\
\text { or death }\end{array}$ & $\begin{array}{l}\text { Observed acute } \\
\text { end-organ injury } \\
\text { or death }\end{array}$ & $\begin{array}{l}\text { Observed acute } \\
\text { end-organ injury } \\
\text { or death }\end{array}$ \\
\hline & Yes n (\%) & No n (\%) & Likelihood ratio & $95 \% \mathrm{CI}$ \\
\hline $\begin{array}{l}\text { Very low risk } \\
<1.5\end{array}$ & $72(3.66)$ & $55,957(8.85)$ & 0.41 & $0.33-0.52$ \\
\hline Low risk 1.5 to 3.0 & $834(42.36)$ & $403,060(63.74)$ & 0.66 & $0.61-0.72$ \\
\hline $\begin{array}{l}\text { Intermediate risk } \\
3.0 \text { to } 5.0\end{array}$ & $408(20.72)$ & $108,085(17.09)$ & 1.21 & $1.09-1.35$ \\
\hline $\begin{array}{l}\text { High risk } 5.0 \text { to } \\
15.0\end{array}$ & $485(24.63)$ & $58,854(9.31)$ & 2.65 & $2.40-2.92$ \\
\hline $\begin{array}{l}\text { Very high risk } \\
>15.0\end{array}$ & $170(8.63)$ & $6,365(1.01)$ & 8.58 & $7.32-10.05$ \\
\hline Total & $1,969(100)$ & $632,321(100)$ & & \\
\hline
\end{tabular}

Figure legends

Figure 1. Receiver operating characteristic curve showing discrimination of the clinical prediction model for maternal end-organ injury or death.

Legend: Outcomes are those arising between 20 weeks' gestation and 42 days after birth, using variables measured pre-pregnancy, and in the index pregnancy prior to 20 weeks' gestation. Analysed is the entire cohort of 634,290 births. C-statistic for Area Under the Curve $=0.68$ (95\% CI 0.66-0.69).

Figure 2. Receiver operating characteristic curve showing the discrimination of the clinical prediction model for maternal end-organ injury or death.

Legend: Outcomes are those arising between 20 weeks' gestation and 42 days after birth using variables measured in the index pregnancy prior to 20 weeks' gestation (2a); the index pregnancy prior to 20 weeks' gestation and pre-pregnancy (2b); the index pregnancy prior to 20 weeks' gestation, pre-pregnancy, and in a previous pregnancy (2c). Analysed is the cohort of 333,435 births among parous women.

Legend, panel a: C-statistic for Area Under the Curve $=0.61$ (95\% CI 0.59-0.63).

Legend, panel b: C-statistic for Area Under the Curve $=0.69$ (95\% CI 0.67-0.70).

Legend, panel c: C-statistic for Area Under the Curve $=0.71$ (95\% CI 0.69-0.73).

Hosted file

DayanCPM-SMM Figure 1 BJOG.pdf available at https://authorea.com/users/385630/articles/ 500909-development-and-internal-validation-of-a-model-predicting-severe-maternal- 
morbidity-using-variables-available-pre-conception-and-in-early-pregnancy-a-populationbased-study

\section{Hosted file}

Dayan CPM-SMM Figure 2a-c BJOG.pdf available at https://authorea.com/users/385630/articles/ 500909-development-and-internal-validation-of-a-model-predicting-severe-maternalmorbidity-using-variables-available-pre-conception-and-in-early-pregnancy-a-populationbased-study 